\title{
The newsvendor problem with pricing: Extensions
}

\author{
Li Yao, Youhua (Frank) Chen*, Houmin Yan ${ }^{1}$ \\ Department of Systems Engineering and Engineering Management, \\ Chinese University of Hong Kong
}

(Received June 12 2006, Accepted July 29 2006)

\begin{abstract}
The newsvendor problem with pricing decision provides an important vehicle for examining how operational problems interacts with marketing issues to influence decision-making at the firm level. This paper considers the newsvendor problem with pricing and its extensions. We try to answer two questions: (1) how to model the price-dependent stochastic demand and (2) how to derive the combined pricing and inventory solutions. We identify a class of demand functions which can result in well behaved profit functions. A demand function can be divided into two parts: the mean demand and random demand. If the part of mean demand has increasing price elasticity (IPE) and the part of random demand has generalized strict increasing failure rate (GSIFR), then the expected profit of the newsvendor is unimodal or quasi-concave. This appears generalizing the existent models in the literature.
\end{abstract}

Keywords: inventory and pricing, newsvendor problem with pricing, stochastic inventory model

\section{Introduction}

As a building block of stochastic inventory theory, the newsvendor problem incorporates the fundamental technique for stochastic decision-making that could be applied to a much broader scope. But in a standard newsvendor problem, the price is assumed to be fixed or exogenously given. Although both the pricing problem in economics and the newsvendor problem in operations management have well-developed results, it is interesting to observe that when considering the combined decision making, there even lacks a consistency in the basic model. More accurately, we have found that the modelling of the price-dependent stochastic demand differs from paper to paper. So to find the appropriate modelling and solution approach for the newsvendor with pricing problem should make meaningful contributions to future research in the related fields.

Bearing such motivations in mind, we address the following questions as the major topics in this research:

- How to properly model the stochastic, price-dependent demand?

- And how to derive the optimal solutions for the newsvendor problem with price and inventory as joint decisions?

For the first question, the model of the stochastic, price-dependent demand can be decomposed into two parts: the mean demand that is contingent on the price, and the stochastic factor that is independent of the price. The most commonly used models assume the mean demand to be a linear function of the price, and to assume the stochastic part to follow a uniform distribution. Different functions are used in different papers. This is especially true on the mean demand part, where the models are most variable.

Inspired by its intrinsic economic nature of the problem, we are able to characterize the mean demand with an interesting property. In particular, we assume that the mean demand has increasing price elasticity (IPE). It can be shown later that this property appears generalizing all the functions that have been used in

\footnotetext{
*E-mail address: yhchen@se.cuhk.edu.hk
} 
existing literature. The economic meaning of IPE is intuitive: as the price gets higher, the market response becomes more sensitive, and hence the supplier has less incentive to raise the price further. Our characterization not only frees us from resorting to specific functions, but also captures the key economic essence. Extending an idea in [9], we need only to assume that the stochastic factor follow a distribution that has generalized strict increasing failure rate (GSIFR), which takes most existent models as special cases. Based on these two properties, we are able to establish a general model without using specific functions.

For the second question, most literature tries to prove the objective function to be concave in the price decision, while we show that the objective function can be convex in price. But it is quasi-concave in price in its admissible range. This guarantees the existence and uniqueness of the optimal solution. Furthermore, we prove two monotone properties between the optimal decisions and the marginal cost. We show that the profit function is always supermodular in the optimal price and the marginal cost, which proves that the optimal price is increasing in the marginal cost. With this result, we are able to demonstrate that the output level is strictly decreasing in the marginal cost.

We now provide a brief literature review. More detailed comparison between previous studies and ours will be provided after we have established our basic results later. [22] is the seminal work to combine pricing with inventory decisions. [11] refines Whitin's work by explicitly modelling the uncertainty in the additive form where the actual demand is the summation of the mean demand and the stochastic factor. Mills shows that the optimal retail price is strictly lower when there is uncertainty than when there is not. [8] presents another demand model: the multiplicative form demand. Under this model the actual demand is given as the multiplication of the mean demand and the stochastic factor. They demonstrate that the optimal retail price is strictly higher when there is uncertainty than when there is not. [14] offers an excellent explanation to justify why the pricing strategy appears to differ depending on how randomness is incorporated into the demand function, because different, implicit assumptions are made about demand variance in the two functional forms.

Both the additive and the multiplicative demand models serve as the two basic functional forms to incorporate demand uncertainty into the pricing problem. Subsequent works on the additive model include [3], [24], [10], [14]. The works on the multiplicative model include [13], [25], [24], [14]. These papers use different assumptions on the demand function and cost structure and none could completely generalize others. The major extensions from single period to multiple periods are [8], [12], [3], [26], [20], [4]. For a complete review on the newsvendor problem with pricing and related topics, we refer to [16] and [14].

Finally, as a building block in supply-chain contract analysis, there are significant extensions for the standard newsvendor problem in the contract literature as well (e.g. [9]); however, in this literature retail price is typically assumed to be fixed or the pricing decision is not a concern, with a few exceptions such as [6], [2]. Though our paper considers a situation where the vendor must commit in advance to a single price for the entire selling season, a number of papers have proposed models of the problem of how a vendor should dynamically adjust the price of a product that can be sold during a fixed amount of time. Examples of this type research include [7], [5], dealing mainly with perishable products such as airplane seats and seasonable items.

The organization of this paper is as follows. We elaborate the newsvendor problem with pricing in Section 2. Section 3 addresses the optimization of the newsvendor problem. Section 4 concludes the paper. For better exposition, we put all the detailed proofs in the Appendix.

\section{The model}

We consider a supplier serving a market with a single product whose demand is price-dependent. The marginal cost of production is a constant $c$, and the objective of the supplier is to choose both the price $p$ and the output-level/order-quantity $q$ to maximize his expected profit $\Pi$.

The demand that the supplier faces, $D(p, \xi)$, is both stochastic and price-dependent, where $\xi$ is a random variable independent of $p$. We assume the expectation of $D$ is specified by a function $y(p)$ for any given price $p$ :

$$
\mathrm{E}_{\xi}[D(p, \xi)]=y(p)
$$


where $y(p)$ is continuous, nonnegative, twice differentiable and is defined on a closed interval $[c, \bar{p}]$, here $\bar{p}$ is the maximum admissible level for $p$, i.e., $d(\bar{p})=0$ for all $p \geq \bar{p}$. Conceivably, $y(p)$ is strictly decreasing in the retail price, i.e., $\frac{d y(p)}{d p}<0$. (Throughout this paper, except explicitly stated otherwise, we will use "increasing" and "decreasing" in the non-strict sense, which means non-decreasing and non-increasing, respectively). Monotonicity of the expected demand in price is satisfied for all regular items, except only special luxury goods exhibiting the Veblen paradox. Under these assumptions, the mean market size is fully known under each price level, hence the uncertainty actually denotes how the demand fluctuates around this expected volume.

Let $e$ denote the price elasticity of $y(p)$, then $e=-\frac{p y^{\prime}(p)}{y(p)}$ by definition. Price elasticity measures the percentage change in demand in response to a percentage change in price. Based on price elasticity we define a class of demand functions as follows.

Definition 1. We say $y(p)$ has an increasing price elasticity (IPE), if

$$
\frac{d e}{d p} \geq 0
$$

The IPE property is intuitive: as the price increases by a certain percentage, the demand decreases by a larger percentage, which makes it less desirable to raise the price further. To the authors' best knowledge, there have been no other papers that have used the notion of IPE in the newsboy context. It can be easily verified that the following examples of $y(p)$, which have been widely used in the literature, satisfy the IPE property:

1. Linear: $y(p)=a(\bar{p}-p), a>0$ ([11]).

2. $y(p)=(1+p)^{-a}, a>1([8])$.

3. $y(p)=p^{-a} \exp (-\lambda p), a>0, \lambda>0$ ([8]).

4. $y(p)$ is log-concave in $p$ ([17]).

5. Iso-elastic: $y(p)=p^{-a}, a>1$ ([14]).

6. $y(p)$ is concave in $p([6],[4])$.

Now we turn to the actual demand incorporating the random component. In the literature two kinds of demand functions, $D(p, \xi)$, have extensively been used: additive and multiplicative forms, which can be integrated as follows:

$$
D(p, \xi)=y_{1}(p) \cdot \xi+y_{2}(p) .
$$

In particular, when $d_{1}(p)=1, D(p, \xi)$ is of additive form, while when $d_{2}(p)=0, D(p, \xi)$ is of multiplicative form. The two models of demand functions generally render separate handling as will be the case here.

We assume that the random variable $\xi$ has a continuous distribution $F(\xi)$ with density $f(\xi)$. It is also reasonable to assume that $F(\xi)$ is invertible and that $f(\xi)$ has a continuous derivative $f^{\prime}(\xi)$. Define the failure rate function of the $\xi$ 's distribution as:

$$
r(\xi)=\frac{f(\xi)}{1-F(\xi)}
$$

and the generalized failure rate function as

$$
g(\xi)=\xi \cdot r(\xi)
$$

Definition 2. We say $\xi$ has generalized strict increasing failure rate (GSIFR) if $g^{\prime}(\xi)>0$ for all $\xi$.

Now we briefly review and compare the relationships among different characterizations of the random distribution, which are subsets of GSIFR class. The strongest condition to ensure GSIFR is to assume $\xi$ having $\log$-concave density $f$. Notice that the following three are equivalent:

(1) $\xi$ has a $\log$-concave density function; 
(2) $\xi$ has a $\mathrm{PF}_{2}$ (Polya frequency function of order 2) density;

(3) $\xi$ has increasing likelihood ratio [19], (p.432-433). Specific examples include: Uniform, Normal (as well as truncated Normal at zero), Exponential, Gamma (with shape parameter $s \geq 1$ ), Beta (with parameters $(r, s)$ being both $\geq 1$ ), and Weibull distribution (with shape parameter $s \geq 1$ ).

A weaker condition to ensure GSIFR is to assume that $\xi$ has a log-concave complement (i.e., $1-F$ is log-concave). This assumption is equivalent to:

(1) $\xi$ has increasing failure rate or

(2) $1-F$ is $\mathrm{PF}_{2}$ ([1], p.25). The log-concave complement class includes the log-concave density class and other distributions such as log-normal with parameter restrictions. In addition, many discrete distributions also have log-concave complements, such as Poisson, Binomial, Hypergeometric and negative Binomial (with shape parameter restrictions) distributions ${ }^{[18]}$.

The GSIFR generalizes the log-concave complement class and provides other flexibilities. For example, the log-concave complement class requires the distributions to have a coefficient of variation of less than one ${ }^{[1]}$ but the GSIFR does not. The GSIFR class includes all Gamma and Weibull distributions but the logconcave complement class requires parameters restrictions ${ }^{[14]}$. The distributions with GSIFR include a broad class enough to incorporate most distributions that would likely be adopted as the distribution for the random factor.

[18] discusses, in a different context, demand distribution functions which are price-independent. He relates properties of log-concave density, log-concave probability function, log-concave complement and monotone convolution ratios. A detailed description on the property of IFR can be found in [1]. Early application of this property in inventory research can be found in [20]. Generalized IFR is discussed in [9] (but in which only the random demand component is considered as the price is fixed).

These relationships can be summarized in the following figure:

$$
\text { GSIFR }\left\{\begin{array} { l } 
{ \text { log-concave complement } } \\
{ \text { or IFR } } \\
{ \text { or } 1 - F \text { is } \mathrm { PF } _ { 2 } } \\
{ \text { Gamma } } \\
{ \text { Weibull } }
\end{array} \left\{\begin{array} { l } 
{ \text { increasing } } \\
{ \text { likelihood ratio } } \\
{ \text { or } f \text { is } \mathrm { PF } _ { 2 } } \\
{ \text { log-normal (subset) } }
\end{array} \left\{\begin{array}{l}
\text { uniform } \\
\text { normal } \\
\text { truncated normal } \\
\text { exponential } \\
\text { beta (subset) } \\
\text { gamma (subset) } \\
\text { weibull (subset) }
\end{array}\right.\right.\right.
$$

Without loss of generality, we set all other costs including goodwill losing and holding costs to be zero. We also assume that any un-sold inventory will have zero salvages value. These assumptions will not impact the qualitative properties of the problem, but can make the expressions more succinct.

We summarize the notations in the following list which include those appearing previously and to appear later.

\begin{tabular}{ll|ll}
\hline$c:$ & the marginal cost & $f:$ & density function of $\xi$ \\
$p:$ & the retail price & $F:$ & cumulative distribution function of $\xi$ \\
$\bar{p}:$ & the maximum admissible price & $r:$ & failure rate function of $\xi$ \\
$q:$ & the output-level/order-quantity & $g:$ & generalized failure rate function of $\xi$ \\
$D:$ & the stochastic demand & $\Pi_{d}, \Pi:$ & riskless and the expected profit, resp. \\
$y(p):$ & the mean value of the demand $y(p)$ & $M R:$ & riskless marginal revenue \\
$e:$ & the price elasticity of $y(p)$ & $\mathrm{E}:$ & the expectation operator \\
$\xi:$ & the random component of $D$ & $\wedge(\vee):$ & the min $(\max )$ operator \\
\hline
\end{tabular}




\section{Joint optimal pricing and ordering decisions}

For ease of exposition, we decompose the analysis into parts: first considering only the deterministic part of demand function and then solving the overall optimization problem. Since focusing on deterministic demand component ignores uncertainty, the resulted optimization problem is called a riskless problem.

\section{Analysis of riskless problem}

Consider the pricing decision without demand uncertainty and define the riskless profit function as:

$$
\Pi_{d}=(p-c) y(p) .
$$

Let $p_{d}$ denote the optimal price that maximizes $\Pi_{d}$. Note that (1) is the typical deterministic pricing problem in the economics context.

Lemma 1. If $y(p)$ has increasing price elasticity, then the riskless profit $\Pi_{d}$ is quasi-concave (or unimodal) in $p$ in the interval $[c, \bar{p}]$. The optimal riskless price $p_{d}$ can be uniquely determined by solving $\frac{d \Pi_{d}}{d p}=0$.

Besides the rigorous mathematical proof, let's see how an IPE demand function leads to Lemma 1. Define the riskless marginal revenue as:

$$
M R=\frac{\partial(p y(p))}{\partial y(p)}
$$

which indicates the revenue increases if one increases the demand $y(p)$ by one unit (or equivalently, from decreasing the price so that demand is increased by one unit). Simple algebra manipulation yields

$$
M R=p\left(1-\frac{1}{e}\right)
$$

Solving $\frac{d \Pi_{d}}{d p}=0$ means letting the marginal revenue equal to the marginal cost:

$$
M R=c .
$$

When the price elasticity $e$ is increasing in $p$ monotonically, the marginal revenue must also be strictly increasing in $p$. Hence the marginal revenue curve can intersect the constant marginal cost curve $c$ at only one point.

\subsection{Optimal solution}

For the rest of this section, the multiplicative form of demand function will be used for mathematical illustration. We provide a sketch of the proof for relevant results related to the additive form in the Appendix.

We assume the mean of $\xi$ to equation (1). The density of demand $D(p, \xi)$ can be expressed as :

$$
\frac{1}{y(p)} f\left(\frac{x}{y(p)}\right), \quad 0 \leq x
$$

The expected profit for any output level $q$ and price $p$ is:

$$
\Pi=\mathrm{E}_{\xi}\{p[q \wedge D(p, \xi)]-c q\} .
$$

The supplier's objective is to choose $\left(p^{*}, q^{*}\right)$ so as to maximize the expected profit $\Pi$ :

$$
\Pi\left(p^{*}, q^{*}\right)=\max _{q, p} \Pi(q, p) .
$$

Use $(a \wedge b)=a-[a-b]^{+}$and rearrange the terms of $\Pi$ : 


$$
\begin{aligned}
\Pi & =\mathrm{E}_{\xi}\left\{(p-c) q-p[q-D(p, \xi)]^{+}\right\} \\
& =(p-c) q-p \int_{0}^{\frac{q}{y(p)}}[q-y(p) t] f(t) d t \\
& =(p-c) q-p y(p) \int_{0}^{\frac{q}{y(p)}} F(t) d t .
\end{aligned}
$$

The last step is done through integration by parts. Let's first fix $p$, then (2) reduces to the standard newsvendor problem without pricing decision and the optimal inventory level $q^{*}$ is given by:

$$
q^{*}=y(p) F^{-1}(\rho)
$$

where $\rho=\frac{p-c}{p}$. Now substitute the above into (2):

$$
\begin{aligned}
\Pi & =y(p)\left[(p-c) F^{-1}(\rho)-p \int_{0}^{F^{-1}(\rho)} F(t) d t\right] \\
& =p y(p) \int_{0}^{F^{-1}(\rho)} t f(t) d t .
\end{aligned}
$$

We are now facing a problem with only one decision variable $p$. Take derivative w.r.t. $p$ :

$$
\frac{\partial \Pi}{\partial p}=p y^{\prime}(p) \int_{0}^{F^{-1}(\rho)} t f(t) d t+y(p)\left[\int_{0}^{F^{-1}(\rho)} t f(t) d t+\frac{c}{p} F^{-1}(\rho)\right] .
$$

The optimal price can be obtained by solving $\frac{\partial \Pi}{\partial p}=0$, but a closed form expression is not obtainable in general. The next theorem guarantees that setting (3) to equal zero is sufficient to determine a unique solution and a one dimensional search will find the optimal $p^{*}$.

Theorem 1. If the mean demand $y(p)$ has IPE and the distribution $F(\xi)$ has GSIFR, then II is quasi-concave in $p$ in the range $[c, \bar{p}]$.

In the literature of newsvendor-pricing problem the existence and uniqueness of the optimal outputlevel/order-quantity and the price have been confined to specific functional forms on both $y(p)$ and the distribution of $\xi$. The following table summarizes the major literature using demand models in the multiplicative form.

Table 1. Demand models in the multiplicative form

\begin{tabular}{lll}
\hline Paper & Mean demand & Random factor distribution \\
\hline Nevins (1966) & linear & normal \\
Zabel (1970) & $p y(p)$ concave in $p$ & uniform, expnential \\
Young (1978) & concave & $\mathrm{PF}_{2}$, log-normal \\
Polatoglu (1991) & linear & exponential \\
Petruzzi and Dada (1999) & power function & increasing failure rate \\
\hline
\end{tabular}

We remark on several papers which were cited previously but have been excluded from the table. [8] concern mainly the qualitative relationship between the optimal retail price when there is uncertainty and that when there is not. However, they do not address issues such as the uniqueness of optimal price level and production/order quantity. As a result they do not need assumptions on $y(p)$ and $f(\xi)$. [25] implicitly assumes the mean demand model to have $e \geq 1$, which satisfies the IPE property, and confines himself to specific stochastic demand functions: uniform or exponential distribution in both the additive and multiplicative form. In analyzing a supply chain contract problem, [2] also use this assumption in dealing with the newsvendor problem. [17] is excluded because it concerns only deterministic demand. 
Table 2. Demand models in the additive form

\begin{tabular}{lll}
\hline Paper & Mead demand & Random factor distribution \\
\hline Mills (1959) & linear & uniform \\
Ernst (1970) & linear & $\mathrm{PF}_{2}$ \\
Thowsen $(1975)^{[20]}$ & linear & $\mathrm{PF}_{2}$ \\
Lau and Lau (1988) & linear & uniform \\
Polatoglu (1991) & linear & uniform \\
Ha (1999) & concave & increasing failure rate \\
Petruzzi and Dada (1999) & linear & increasing failure rate \\
\hline
\end{tabular}

The following table summarizes the major literature using demand model in the additive form.

The references appearing in the above two tables and preceding remarks are mainly taken from the review paper by [14], with additional references which we have identified elsewhere. These results can all be viewed as special cases of Theorem 1. The articulation of the theorem can thus be viewed as a generalization of the existing literature, and our description of $y(p)$ and $f$ in terms of certain properties rather than specific functions may serve as the modeling framework for other related research.

It is important to explain why [4] ( a multiple period model) has been excluded. Federgruen and Heching's model differs significantly from other multi-period models: they assume full-backlogging while others consider lost-sales ${ }^{[20,26]}$. So the revenue part in Federgruen and Heching is $p * D$ while in all other papers it is truncated. They propose a model with concave mean demand (in price) and without specifying the stochastic part. Though the concave mean demand satisfies the IPE property, the random component in their model need not take the additive or multiplicative from. They can get around specifying $f$ because the expected revenue in each period is exactly the mean demand multiplied by price under the assumptions of backordering and zero leadtime, while in the newsvendor setting, the revenue is truncated. [23] shows that if considering the newsvendor version of Federgruen and Heching, their demand function can be even further extended to a broader class.

\subsection{Impact of the Marginal Cost}

Now let's consider the impact of the magnitude of the marginal cost $c$ on the supplier's decisions. We first calculate the cross partial as:

$$
\frac{\partial^{2} \Pi}{\partial p \partial c}=-\left[y^{\prime}(p) F^{-1}(\rho)+\frac{c}{p^{2}} y(p) \frac{1}{f\left(F^{-1}(\rho)\right)}\right] .
$$

In the riskless case, the supplier will increase the price $p$ in response to a higher marginal cost and the output level will be lowered accordingly. We can show that such result also holds in the stochastic case:

Theorem 2. Monotone property between $p^{*}$ and $c$.

If the mean demand $y(p)$ has IPE and the distribution F has GSIFR,

1. If $\frac{\partial^{2} \Pi}{\partial p \partial c} \leq 0$, then there must be $\frac{\partial \Pi}{\partial p}>0$. So the optimal price $p^{*}$ cannot be found in the area where $\Pi$ is submodular in $(p, c)$.

2. In other words, $\Pi$ is strictly supermodular in $\left(p^{*}, c\right)$, hence the optimal price $p^{*}$ is strictly increasing in $c$.

As $p^{*}$ will be higher as $c$ is increased, how will the output level be adjusted? The mean demand will be smaller under a higher price, but its effect on the critical ratio $\frac{p-c}{p}$ is rather unclear. Fortunately we have also found certain monotone property for this issue. Let's first define $\rho^{*}=\frac{p^{*}-c}{p^{*}}$, i.e., it is the critical factile at the optimal decision.

Theorem 3. Monotone property between $q^{*}$ and $c$.

If the mean demand $y(p)$ has IPE and the distribution F has GSIFR,

1. The optimal critical fractile $\rho^{*}$ is decreasing in c, i.e., $\frac{d \rho^{*}}{d c} \leq 0$. and the equality holds when $y(p)$ has iso-elasticity. 


\section{The optimal output level $q^{*}$ is strictly decreasing in $c$.}

Now we can conclude that under the newsvendor pricing setting, an increased marginal cost will lead to a higher price, which in turn causes a smaller market demand and a smaller output level.

There are two papers concerning this impact of marginal cost in the literature. [9] studies the standard newsvendor model where the price is fixed rather than is a decision variable. There he finds the monotone property between $q$ and $c$ just as stated in Theorem 3. [6] finds similar results for the additive model where he assumes $y(p)$ to be a concave function which has been shown to belong to the IPE classes. Hence Theorem 2 and 3 justify existing results, and further generalize them.

\section{Conclusion}

The major effort of this research is to establish an appropriate general demand model that has appealing and intuitive economical interpretation yet unifies the existent models. With this model we have shown how the newsvendor problem with joint pricing and output/order decision can be solved without using specific demand functions. We hope this result will provide a good framework for future research on related problems, such as the multi-retailer problem with competitive pricing and supply-chain contract analysis. Some progresses have been made towards these directions, e.g., [23] extends the model to a multi-retailer horizontal supply chain competing in prices and a vertical supply chain coordinating the price and output level through contract design.

\section{References}

[1] R. E. Barlow, F. Proschan. Mathematical Theory of Reliability, SIAM Classics in Applied Mathematics series. Philadelphia, 1996.

[2] H. Emmons, S. Gilbert. Return Policies in Pricing and Inventory Decisions for Catalogue Goods. Management Science, 1998, 44(2): 276-283.

[3] R. L. Ernst. A Linear Inventory Model of a Monopolistic Firm. Ph.D. Thesis, University of California Berkeley, 1970.

[4] A. Federgruen, A. Heching. Combined pricing and inventory control under uncertainty. Operations Research, 1999, 47(3): 454-475.

[5] Y. Feng, V. Ryzin, B. Xiao. Optimal policies of yield management with multiple predetermined prices. Operations Research, 2000.

[6] A. Ha. Supplier-buyer contracting: Asymmetric cost information and cut-off level policy for buyer participation, 1999. Working paper in Yale School of Management.

[7] S. Kalish. Monopoly pricing with dynamic demand and producion cost. Marketing SCI, 1983, 2: 135-159.

[8] S. Karlin, C. R. Carr. Studies in Applied Probability and Management Science. Stanford Press, 1962.

[9] M. A. Lariviere. Supply chain contracting and coordination with stochastic demand. in: Quantitative Models for Supply Chain Management, 1998.

[10] H. Lau, S. Lau. The Newsvendor problem with Price-Dependent demand distribution. IIE Transactions, 1988, 20(2): 168-175.

[11] E. S. Mills. Uncertainty and price theory. Quarterly Journal of Economics, 1959, 73(1): 116-130.

[12] E. S. Mills. in: Price, Output and Inventory Policy (J. Wiley, Sons, eds.), 1962.

[13] A. J. Nevins. Some effects of uncertainty:Simulation. Quarterly Journal of Economics, 1966, 80: $73-87$.

[14] N. Petruzzi, M. Dada. Pricing and the newsvendor problem: A review with extensions. Operations Research, 1999, 47(2): 183-194.

[15] L. H. Polatoglu. Optimal order quantity and pricing decisions in single period inventory systems. International Journal of Production Economics, 1991, 23: 175-185.

[16] E. L. Porteus. Stochastic inventory theory. in: Handbooks in Operations Research and Management Science, vol. 4 (G. L. Nemhauser, A. H. G. R. Kana, eds.), North-Holland, 1990, chap. 12, 605-652.

[17] D. Rosenberg. Optimal price-inventory decisions: Profit vs. ROII. IIE Transactions, 1991, 23(1): 17-22.

[18] K. Rosling. Inventory cost rate functions with nonlinear shortage costs, 2000. Working paper in Department of Industrial Engineering of Vaxjo University.

[19] S. Ross. Stochastic Processes, 2nd edn. New York, 1996. 
[20] G. T. Thowsen. A dynamic, nonstationary inventory problem for a price/ quantity setting firm. Naval Research Logistics Quarterly, 1975, 22: 461-476.

[21] D. M. Topkis. Minimizing a submodular function on a lattice. Operations Research, 1978, 26(2): 305-321.

[22] T. M. Whitin. Inventory control and price theory. Management Science, 1955, 2: 61-68.

[23] L. Yao. Supply Chain Modeling: Pricing, Contracts and Coordination. Ph.D. Thesis, Chinese University of Hong Kong, 2002.

[24] L. Young. Price, inventory and the structure of uncertain demand. New Zealand J. Oper. Res., 1978, 6: 157-177.

[25] E. Zabel. Monopoly and uncertainty. Review of Economics Studies, 1970, 37: 205-219.

[26] E. Zabel. Multiperiod monopoly under uncertainty. Journal of Economic Theory, 1972, 5: 524-536.

\section{A Appendix}

\section{A.1 Proof of Lemma 1.}

Since $e=-\frac{p y^{\prime}(p)}{y(p)}$,

$$
\frac{d e}{d p}=-\frac{\left[p y^{\prime \prime}(p)+y^{\prime}(p)\right] y(p)-p\left(y^{\prime}(p)\right)^{2}}{y^{2}(p)} .
$$

Since $\frac{d e}{d p} \geq 0$ by assumption, rearranging terms yields:

$$
y^{\prime \prime}(p) \leq\left[\frac{p y^{\prime}(p)}{y(p)}-1\right] \frac{y^{\prime}(p)}{p}
$$

Now take derivative of $\Pi_{d}$ w.r.t. $p$ :

$$
\frac{d \Pi_{d}}{d p}=y^{\prime}(p)(p-c)+y(p)
$$

Since $\left.\frac{d \Pi_{d}}{d p}\right|_{p=c}=y(p)>0$ and $\left.\frac{\Pi_{d}}{d p}\right|_{p=\bar{p}}=y^{\prime}(\bar{p})(\bar{p}-c)<0, \frac{d \Pi_{d}}{d p}=0$ has at least one solution as long as it is continuous. Take the second derivative:

$$
\frac{d^{2} \Pi_{d}}{d p^{2}}=y^{\prime \prime}(p)(p-c)+2 y^{\prime}(p)
$$

Substitute (5) into the above:

$$
\begin{aligned}
\frac{d^{2} \Pi_{d}}{d p^{2}} & \leq(p-c)\left[\frac{p y^{\prime}(p)}{y(p)}-1\right] \frac{y^{\prime}(p)}{p}+2 y^{\prime}(p) \\
& =\frac{y^{\prime}(p)}{p}\left[p \frac{(p-c) y^{\prime}(p)}{y(p)}+p+c\right] .
\end{aligned}
$$

Because $\frac{d \Pi_{d}}{d p}=0$ implies $\frac{(p-c) y^{\prime}(p)}{y(p)}=-1$,

$$
\left.\frac{d^{2} \Pi_{d}}{d p^{2}}\right|_{\frac{d \Pi_{d}}{d p}=0} \leq \frac{y^{\prime}(p)}{p}[-p+p+c]<0 .
$$

Hence $\Pi_{d}$ is quasi-concave in $p$, and $\frac{d \Pi_{d}}{d p}=0$ has only one unique solution $p_{d}$. 


\section{A.2 Proof of Theorem 1.}

We first show the result by assuming the demand function to take the multiplicative form. From (3), the second derivative of $\Pi$ w.r.t. $p$ is:

$$
\begin{aligned}
\frac{\partial^{2} \Pi}{\partial p^{2}}= & y^{\prime \prime}(p) p \int_{0}^{F^{-1}(\rho)} t f(t) d t+2 y^{\prime}(p)\left[\int_{0}^{F^{-1}(\rho)} t f(t) d t+F^{-1}(\rho) \frac{c}{p}\right] \\
& +y(p) \frac{1}{f\left(F^{-1}(\rho)\right)} \frac{c^{2}}{p^{3}} .
\end{aligned}
$$

It is easy to verify that $\left.\frac{\partial \Pi}{\partial p}\right|_{p=c}=0$ and $\left.\frac{\partial^{2} \Pi}{\partial p^{2}}\right|_{p=c}=y(c) \frac{1}{f(0)} \frac{1}{c}>0$. This means that $\frac{\partial \Pi}{\partial p}>0$ for sufficiently small $p>c$. Also $\left.\frac{\partial \Pi}{\partial p}\right|_{p=\bar{p}}=\bar{p} y^{\prime}(\bar{p}) \int_{0}^{F^{-1}(\rho)} F(t) d t<0$, so $\frac{\partial \Pi}{\partial p}=0$ must have at least one solution on $(c, \bar{p})$ as long as it is continuous. To prove the solution is also unique, we need to prove $\left.\frac{\partial^{2} \Pi}{\partial p^{2}}\right|_{\frac{\partial \Pi}{\partial p}=0}<0$.

Under the assumption that $y(p)$ is IPE, apply (5) and substitute $y^{\prime \prime}(p)$ :

$$
\begin{aligned}
\frac{\partial^{2} \Pi}{\partial p^{2}} \leq & {\left[\frac{p y^{\prime}(p)}{y(p)}-1\right] y^{\prime}(p) \int_{0}^{F^{-1}(\rho)} t f(t) d t } \\
& +2 y^{\prime}(p)\left[\int_{0}^{F^{-1}(\rho)} t f(t) d t+F^{-1}(\rho) \frac{c}{p}\right] \\
& +y(p) \frac{1}{f\left(F^{-1}(\rho)\right)} \frac{c^{2}}{p^{3}} \\
= & y^{\prime}(p)\left[\left(\frac{p y^{\prime}(p)}{y(p)}+1\right) \int_{0}^{F^{-1}(\rho)} t f(t) d t+2 F^{-1}(\rho) \frac{c}{p}\right] \\
& +y(p) \frac{1}{f\left(F^{-1}(\rho)\right)} \frac{c^{2}}{p^{3}} .
\end{aligned}
$$

If $\frac{\partial \Pi}{\partial p}=0$, from (3) we have:

$$
\left(\frac{p y^{\prime}(p)}{y(p)}+1\right) \int_{0}^{F^{-1}(\rho)} t f(t) d t=-F^{-1}(\rho) \frac{c}{p}
$$

Substitute into $\frac{\partial^{2} \Pi}{\partial p^{2}}$ :

$$
\begin{aligned}
\left.\frac{\partial^{2} \Pi}{\partial p^{2}}\right|_{\frac{\partial \Pi}{\partial p}=0} & \leq y^{\prime}(p) F^{-1}(\rho) \frac{c}{p}+y(p) \frac{1}{f\left(F^{-1}(\rho)\right)} \frac{c^{2}}{p^{3}} \\
& =\frac{c}{p^{2}}\left[y^{\prime}(p) p F^{-1}(\rho)+y(p) \frac{1}{f\left(F^{-1}(\rho)\right)} \frac{c}{p}\right] \\
& =\frac{c}{p^{2} \int_{0}^{F^{-1}(\rho)} t f(t) d t}\left[y^{\prime}(p) p \int_{0}^{F^{-1}(\rho)} t f(t) d t F^{-1}(\rho)+y(p) \frac{\int_{0}^{F^{-1}(\rho)} t f(t) d t}{f\left(F^{-1}(\rho)\right)} \frac{c}{p}\right] .
\end{aligned}
$$

Again from (3):

$$
y^{\prime}(p) p \int_{0}^{F^{-1}(\rho)} t f(t) d t=-y(p)\left[\int_{0}^{F^{-1}(\rho)} t f(t) d t+F^{-1}(\rho) \frac{c}{p}\right] .
$$

Substitute into $\frac{\partial^{2} \Pi}{\partial p^{2}}$ :

$$
\left.\frac{\partial^{2} \Pi}{\partial p^{2}}\right|_{\frac{\partial \Pi}{\partial p}=0} \leq \frac{c y(p)}{p^{2} \int_{0}^{F^{-1}(\rho)} t f(t) d t}\left\{-\left[\int_{0}^{F^{-1}(\rho)} t f(t) d t+F^{-1}(\rho) \frac{c}{p}\right] F^{-1}(\rho)+\frac{\int_{0}^{F^{-1}(\rho)} t f(t) d t}{f\left(F^{-1}(\rho)\right)} \frac{c}{p}\right\} .
$$


Now we only need prove that the terms inside the \{\} is negative. To transform those terms into a more convenient form, let $x=F^{-1}(\rho)$, then $x>0$ and $\rho=F(x)$. Rewrite the terms inside the curly bracket as a function of $x$ :

$$
\begin{aligned}
A(x) & =-\left[\int_{0}^{x} t f(t) d t+(1-F(x)) x\right] x+\frac{\int_{0}^{x} t f(t) d t}{f(x)}(1-F(x)) \\
& =-\left[\int_{0}^{x} t f(t) d t+(1-F(x)) x\right] x+\frac{\int_{0}^{x} t f(t) d t}{r(x)} .
\end{aligned}
$$

It is easy to verify $A(0)=0$. Then $A^{\prime}(x)<0$ will guarantee $A(x)<0$ for all $x>0$. It can be shown:

$$
\begin{aligned}
A^{\prime}(x) & =-(1-F(x)) x-\left[\int_{0}^{x} t f(t) d t+(1-F(x)) x\right]+\frac{x f(x) r(x)-r^{\prime}(x) \int_{0}^{x} t f(t) d t}{r^{2}(x)} \\
& =-2(1-F(x)) x-\int_{0}^{x} t f(t) d t+\frac{x f(x)}{r(x)}-\frac{r^{\prime}(x) \int_{0}^{x} t f(t) d t}{r^{2}(x)} \\
& =-(1-F(x)) x-\int_{0}^{x} t f(t) d t+\left(-\frac{x r^{\prime}(x)}{r(x)}\right) \frac{\int_{0}^{x} t f(t) d t}{x r(x)}
\end{aligned}
$$

As we have assumed, $\xi$ has GSIFR. It is easy to show this will lead to:

$$
-\frac{x r^{\prime}(x)}{r(x)}<1
$$

So:

$$
A^{\prime}(x)<-(1-F(x)) x-\int_{0}^{x} t f(t) d t+\frac{\int_{0}^{x} t f(t) d t}{x r(x)}
$$

Define

$$
B(x)=-(1-F(x)) x-\int_{0}^{x} t f(t) d t+\frac{\int_{0}^{x} t f(t) d t}{x r(x)} .
$$

Now we want to prove that $B(x)<0$. Since:

$$
\lim _{x \rightarrow 0} \frac{\int_{0}^{x} t f(t) d t}{x r(x)}=\lim _{x \rightarrow 0} \frac{x f(x)}{r(0)}=0
$$

then obviously:

$$
\lim _{x \rightarrow 0} B(x)=0
$$

The derivative of $B(x)$ is:

$$
\begin{aligned}
B^{\prime}(x) & =-(1-F(x))+\frac{x^{2} f(x) r(x)-(x r(x))^{\prime} \int_{0}^{x} t f(t) d t}{(x r(x))^{2}} \\
& =-\frac{(x r(x))^{\prime} \int_{0}^{x} t f(t) d t}{(x r(x))^{2}} \\
& =-\frac{g^{\prime}(x) \int_{0}^{x} t f(t) d t}{g^{2}(x)} .
\end{aligned}
$$

Again according to the GSIFR assumption, $g^{\prime}(x)>0$. Then going one by one, $B^{\prime}(x)<0, B(x)<$ $0, A^{\prime}(x)<0$ and $A(x)<0$ for $x>0$.

Now we can conclude 


$$
\left.\frac{\partial^{2} \Pi}{\partial p^{2}}\right|_{\frac{\partial \Pi}{\partial p}=0}<0
$$

So $\Pi$ is quasi-concave in $p$ and $\frac{\partial \Pi}{\partial p}=0$ has a unique solution.

Now we highlight the proof for the additive form of demand function. The demand now has the form:

$$
D(p, \xi)=y(p)+\xi
$$

The objective function in (2) now becomes:

$$
\Pi=(p-c) q-p \int_{0}^{q-y(p)}[q-y(p)-t] f(t) d t .
$$

First fix $p$, solve the optimal output level $q^{*}$ as $q^{*}=y(p)+F^{-1}(\rho)$, and substitute into the above:

$$
\Pi=(p-c) y(p)+p \int_{0}^{F^{-1}(\rho)} t f(t) d t .
$$

Take derivative w.r.t. $p$ :

$$
\begin{aligned}
\frac{\partial \Pi}{\partial p} & =(p-c) y^{\prime}(p)+y(p)+\left[\int_{0}^{F^{-1}(\rho)} t f(t) d t+\frac{c}{p} F^{-1}(\rho)\right], \\
\frac{\partial^{2} \Pi}{\partial p^{2}} & =(p-c) y^{\prime \prime}(p)+2 y^{\prime}(p)+\frac{1}{f\left(F^{-1}(\rho)\right)} \frac{c^{2}}{p^{3}} .
\end{aligned}
$$

Similar as the multiplicative model, we will need to prove $\left.\frac{\partial^{2} \Pi}{\partial p^{2}}\right|_{\frac{\partial \Pi}{\partial p}=0}<0$. Applying the IPE property, substituting and rearranging terms yields:

$$
\left.\frac{\partial^{2} \Pi}{\partial p^{2}}\right|_{\frac{\partial \Pi}{\partial p}=0}<\frac{c}{p^{2} \rho}\left[-\left(\int_{0}^{F^{-1}(\rho)} t f(t) d t+\frac{c}{p} F^{-1}(\rho)\right)+\frac{1}{f\left(F^{-1}(\rho)\right)} \frac{c}{p} \rho\right]
$$

Now let $x=F^{-1}(\rho)$ we can transform the terms within [] into a more convenient function in $x$. Make use of the proves in the multiplicative model we can show that this function is negative, so:

$$
\left.\frac{\partial^{2} \Pi}{\partial p^{2}}\right|_{\frac{\partial \Pi}{\partial p}=0}<0 .
$$

All the technical details are contained in [23].

\section{A.3 Proof of theorem 2}

\section{A.3.1 Part 1}

If $\frac{\partial^{2} \Pi}{\partial p \partial c} \leq 0$, from (4):

$$
y^{\prime}(p) \geq-\frac{c}{p^{2}} y(p) \frac{1}{f\left(F^{-1}(\rho)\right) \cdot F^{-1}(\rho)} .
$$

Substitute into (3):

$$
\begin{aligned}
\frac{\partial \Pi}{\partial p} & \geq y(p)\left[-\frac{c}{p} \frac{\int_{0}^{F^{-1}(\rho)} t f(t) d t}{f\left(F^{-1}(\rho)\right) \cdot F^{-1}(\rho)}+\int_{0}^{F^{-1}(\rho)} t f(t) d t+\frac{c}{p} F^{-1}(\rho)\right] \\
& =\frac{-y(p)}{f\left(F^{-1}(\rho)\right)}\left\{\frac{c}{p} \frac{\int_{0}^{F^{-1}(\rho)} t f(t) d t}{f\left(F^{-1}(\rho)\right)}-\left[\int_{0}^{F^{-1}(\rho)} t f(t) d t+\frac{c}{p} F^{-1}(\rho)\right] f\left(F^{-1}(\rho)\right)\right\} .
\end{aligned}
$$


Since $y(p)>0$, what we need is to prove the terms in the bracket is negative. Similar as in the proof of Theorem 1, we use some technique to transform those terms into a more convenient form. Let $x=F^{-1}(\rho)$, then $x>0$ and $\rho=F(x)$. we can now rewrite the terms in the bracket as a function of $x$ :

$$
A(x)=\frac{\int_{0}^{x} t f(t) d t}{f(x)}(1-F(x))-\left[\int_{0}^{x} t f(t) d t+(1-F(x)) x\right] x
$$

Notice the above is the same as equation (8), which has been proved to be negative. Then we can conclude $\left.\frac{\partial \Pi}{\partial p}\right|_{\frac{\partial^{2} \Pi}{\partial p \partial c} \leq 0}>0$ for all $p$, which means that $\frac{\partial \Pi}{\partial p}=0$ has no solution if $\Pi$ is submodular in $(p, c)$.

\section{A.3.2 Part 2}

Now we have seen that $\Pi$ is strictly supermodular in $(p, c)$ when $\frac{\partial \Pi}{\partial p}=0$. From Theorem 6.3 of [21], and noticing that maximizing a supermodular function is equivalent to minimizing a submodular function, we can conclude that the optimal price $p^{*}$ is strictly increasing in $c$.

\section{A.4 Proof of Theorem 3}

\section{A.4.1 Part 1}

For better exposition, in the proof of Theorem 3 all $p$ is actually $p^{*}$. We intentionally omit the superscript that implies the optimal solution.

From the definition of $\rho$ :

$$
\frac{d \rho}{d c}=\frac{c \frac{d p}{d c}-p}{p^{2}}
$$

What we need to prove is that the numerator is positive. Since the optimal $p$ is obtained by $\frac{\partial \Pi}{\partial p}=0$, make use of the chain rule and take derivative w.r.t $c$ :

$$
\frac{\partial^{2} \Pi}{\partial p^{2}} \cdot \frac{d p}{d c}+\frac{\partial^{2} \Pi}{\partial p \partial c}=0
$$

Further rearranging terms yields:

$$
\left(c \frac{d p}{d c}-p\right) \frac{\partial^{2} \Pi}{\partial p \partial c}=p \cdot \frac{d p}{d c} \cdot\left(\frac{\partial^{2} \Pi}{\partial p^{2}}+\frac{c}{p} \frac{\partial^{2} \Pi}{\partial p \partial c}\right) .
$$

From theorem 2, we know:

$$
\left.\frac{d p}{d c}\right|_{p=p^{*}}>0, \quad \text { and }\left.\quad \frac{\partial^{2} \Pi}{\partial p \partial c}\right|_{p=p^{*}}>0
$$

Hence $c \frac{d p}{d c}-p$ has the same sign with the term $\frac{\partial^{2} \Pi}{\partial p^{2}}+\frac{c}{p} \frac{\partial^{2} \Pi}{\partial p \partial c}$. As $\frac{\partial^{2} \Pi}{\partial p^{2}}$ and $\frac{\partial^{2} \Pi}{\partial p \partial c}$ are derived in equation (4) and (6) respectively:

$$
\frac{\partial^{2} \Pi}{\partial p^{2}}+\frac{c}{p} \frac{\partial^{2} \Pi}{\partial p \partial c}=y^{\prime \prime}(p) p \int_{0}^{F^{-1}(\rho)} t f(t) d t+y^{\prime}(p)\left[2 \int_{0}^{F^{-1}(\rho)} t f(t) d t+F^{-1}(\rho) \frac{c}{p}\right] .
$$

As we have assumed that $y(p)$ has IPE, substitute equation (5) into the above:

$$
\begin{aligned}
\frac{\partial^{2} \Pi}{\partial p^{2}}+\frac{c}{p} \frac{\partial^{2} \Pi}{\partial p \partial c} & \leq\left\{\left[\frac{p y^{\prime}(p)}{y(p)}-1\right] \int_{0}^{F^{-1}(\rho)} t f(t) d t+2 \int_{0}^{F^{-1}(\rho)} t f(t) d t+F^{-1}(\rho) \frac{c}{p}\right\} y^{\prime}(p) \\
& =\left\{\left[\frac{p y^{\prime}(p)}{y(p)}+1\right] \int_{0}^{F^{-1}(\rho)} t f(t) d t+F^{-1}(\rho) \frac{c}{p}\right\} y^{\prime}(p) .
\end{aligned}
$$



gives:

Remember here $p$ represents $p^{*}$ which is obtained by $\frac{\partial \Pi}{\partial p}=0$, rearranging the terms in equation (3)

$$
\left[\frac{p y^{\prime}(p)}{y(p)}+1\right] \int_{0}^{F^{-1}(\rho)} t f(t) d t+F^{-1}(\rho) \frac{c}{p}=0,
$$

so $\frac{\partial^{2} \Pi}{\partial p^{2}}+\left.\frac{c}{p} \frac{\partial^{2} \Pi}{\partial p \partial c}\right|_{p=p^{*}} \leq 0$, and $\left.\left(c \frac{d p}{d c}-p\right)\right|_{p=p^{*}} \leq 0$ as well. Notice the equality holds when the equality holds in equation (5), which is the case when $y(p)$ has iso-elasticity.

Now we can conclude $\left.\frac{d \rho}{d c}\right|_{p=p^{*}} \leq 0$, with the equality holds when $y(p)$ has iso-elasticity.

\section{A.4.2 Part 2}

Since $q=y(p) F^{-1}(\rho)$,

$$
\frac{d q}{d c}=y^{\prime}(p) F^{-1}(\rho) \frac{d p}{d c}+y(p) \frac{1}{f\left(F^{-1}(\rho)\right)} \frac{d \rho}{d c} .
$$

From the previous part we know the second term on the r.h.s. is non-positive. For the first term on r.h.s., since $y^{\prime}(p)<0$ and $\left.\frac{d p}{d c}\right|_{p=p^{*}}>0$, it is strictly negative. Hence $\frac{d q^{*}}{d c}<0$. 